\title{
The Dendritic Differentiation of Purkinje Neurons: Unsolved Mystery in Formation of Unique Dendrites
}

\author{
Masahiko Tanaka
}

Published online: 12 July 2014

(C) Springer Science+Business Media New York 2014

Purkinje neurons play a central role in cerebellar functions. For Purkinje neurons to play such a role, the unique morphology of their dendrites is important. Purkinje neurons have the most elaborate dendritic trees among neurons in the brain (Fig. 1). They extend long proximal dendrites towards the pial surface. These dendrites branch extensively to form numerous synapses with parallel fibers, axons of granule cells. Purkinje dendrites are formed in a plane that is oriented perpendicular to the long axis of the cerebellum. Such unique dendritic trees differentiate over several postnatal weeks in rodents [1]. To date, many studies have investigated the cellular and molecular mechanisms of the dendritic differentiation of Purkinje neurons [2, 3]. Although those studies identified a number of molecules involved in Purkinje dendrite differentiation, several important aspects concerning differentiation of the unique dendrites remain unclear. This editorial highlights these aspects of the dendritic differentiation of Purkinje neurons (Fig. 2).

\section{Extensive Branching}

The most notable feature of Purkinje dendrites is their extensive branching. What is the mechanism leading to it? What about Purkinje dendrites differs from dendrites of other neurons? Although we cannot yet answer such questions sufficiently, I would like to mention two lines of recent progress in investigations of the molecular mechanisms of the dendritic branching of Purkinje neurons.

M. Tanaka $(\bowtie)$

Department of Cellular Biophysics, Graduate School of

Pharmaceutical Sciences, Nagoya City University,

Nagoya 467-8603, Japan

e-mail:mtanaka@phar.nagoya-cu.ac.jp
Intracellular calcium is an important regulator of the dendritic differentiation of many types of neuron [4]. Purkinje neurons express many types of calcium channel including voltage-gated calcium channels (VGCCs) and intracellular calcium release channels. Although knockout mice deficient in several types of VGCC have been generated, abnormalities in the morphology of Purkinje dendrites of these knockout mice have not been reported, except that Purkinje dendrites of $\mathrm{Ca}_{\mathrm{V}} 2.1(\alpha 1 \mathrm{~A})$ knockout mice appeared to be shorter than those of the wild-type [5]. Thus, no or only mild phenotypes were observed in the dendritic differentiation of Purkinje neurons in these knockout mice, despite the expected importance of VGCCs. However, this might be due to the redundancy of VGCCs. From this viewpoint, it would be interesting to examine the effects of double or triple knockout/ knockdown of VGCCs on the dendritic differentiation of Purkinje neurons.

Regarding intracellular calcium release channels, Purkinje neurons exhibited reduced dendritic branching in knockout mice deficient in inositol 1,4,5-trisphosphate receptor $\left(\mathrm{IP}_{3} \mathrm{R}\right)$ type 1 [6]. However, these abnormalities are thought to be due to a deficiency in $\mathrm{IP}_{3} \mathrm{R} 1$ expressed by granule cells. The production of brain-derived neurotrophic factor (BDNF) is dependent on $\mathrm{IP}_{3} \mathrm{R} 1$ in granule cells, and BDNF released from granule cells has been suggested to promote dendritic branching of Purkinje neurons. A recent knockdown study reported that ryanodine receptor (RyR), another class of intracellular calcium release channel, is also involved in the dendritic branching of cultured Purkinje neurons [7]. Interestingly, not only RyR1 expressed by Purkinje neurons but also RyR2 expressed by granule cells is involved in dendritic branching of Purkinje neurons. Also, BDNF released from granule cells promotes the dendritic branching of Purkinje neurons downstream of RyR2 expressed by granule cells, suggesting similar roles of $\mathrm{IP}_{3} \mathrm{R} 1$ and RyR2 in granule cells (production or release of BDNF). Considering this hypothesis, 


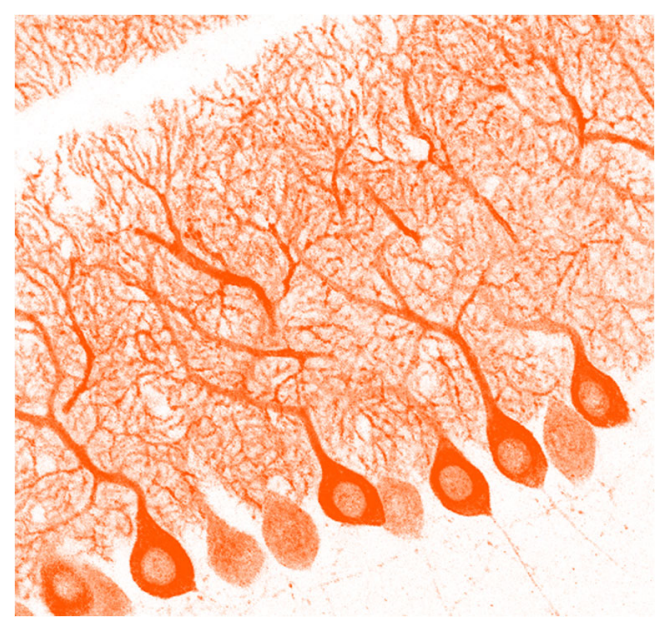

Fig. 1 Purkinje neurons visualized by immunohistochemistry against $\mathrm{IP}_{3} \mathrm{R} 1$ in a cerebellar slice of a 14-day-old mouse

it would be interesting to examine the effects of double knockout/knockdown of $\mathrm{IP}_{3} \mathrm{R} 1$ and RyR2 in granule cells on the dendritic differentiation of Purkinje neurons.

The second line of recent progress concerns the actin cytoskeleton. For dendrites to branch, cytoskeletons need to be reorganized. The rate-limiting step in actin filament formation is actin nucleation, the assembly of actin nuclei. CordonBleu (Cobl) is the WH2 domain-based actin nucleator [8]. A recent study using cerebellar slice cultures reported that the dendritic branching of Purkinje neurons requires the interaction between Cobl and the F-actin binding protein Abp1 [9]. Since Cobl is highly expressed in Purkinje neurons, the Cobl/Abp1 interaction may promote reorganization of the actin cytoskeleton in differentiating Purkinje dendrites, leading to their extensive branching. It would be interesting to examine the involvement of other actin nucleators such as the Arp $2 / 3$ complex, formins, and Spire in the dendritic branching of Purkinje neurons [9].

\section{Retraction of Dendrites}

One of the characteristic aspects of the formation of Purkinje dendrites is their morphological changes during the differentiation process. The morphology of Purkinje dendrites changes markedly during postnatal cerebellar development [10-12]. In the first postnatal week in mice and rats, Purkinje neurons change their morphology from "fusiform/bipolar" to "stellate with disoriented dendrites", which means a morphology with multiple short processes extending in random orientations. In the second postnatal week, Purkinje neurons have only one primary dendrite extending in one direction (towards the pial surface). Obviously, retraction of some dendrites occurs during the latter transition process. In fact, the retraction of some dendrites was demonstrated to occur by live imaging of cultured Purkinje neurons $[13,14]$. Thus, the characteristic morphology of Purkinje dendrites is formed not only through extension, but also through retraction.

Then, what is the mechanism underlying the retraction of dendrites? Several studies have reported such mechanisms. Protein kinase $\mathrm{C}$ (PKC) $\gamma$ and $\mathrm{PKC} \mu$ (PKD1) were shown to be negative regulators of dendritic differentiation which induce the retraction of dendrites in Purkinje neurons $[2,15]$. Interestingly, it was demonstrated that retraction is triggered by contact between growing dendrites using time-lapse imaging of cultured Purkinje neurons [15]. Recent studies elucidated the involvement of several membrane proteins in the retraction of Purkinje dendrites. A study using knockout and transgenic mice showed that Nogo-A is a negative regulator of the dendritic differentiation of Purkinje neurons [16]. Since the Nogo receptor (NgR1) is localized in parallel fiber terminals, it remains to be elucidated how Nogo- $\mathrm{A} / \mathrm{NgR} 1$ signaling influences Purkinje dendrites. Furthermore, protocadherins [17] and cell-autonomous Slit2/Robo2 signaling [18] were reported to mediate self-avoidance of differentiating Purkinje
Fig. 2 Schematic representation of the molecules described in this editorial

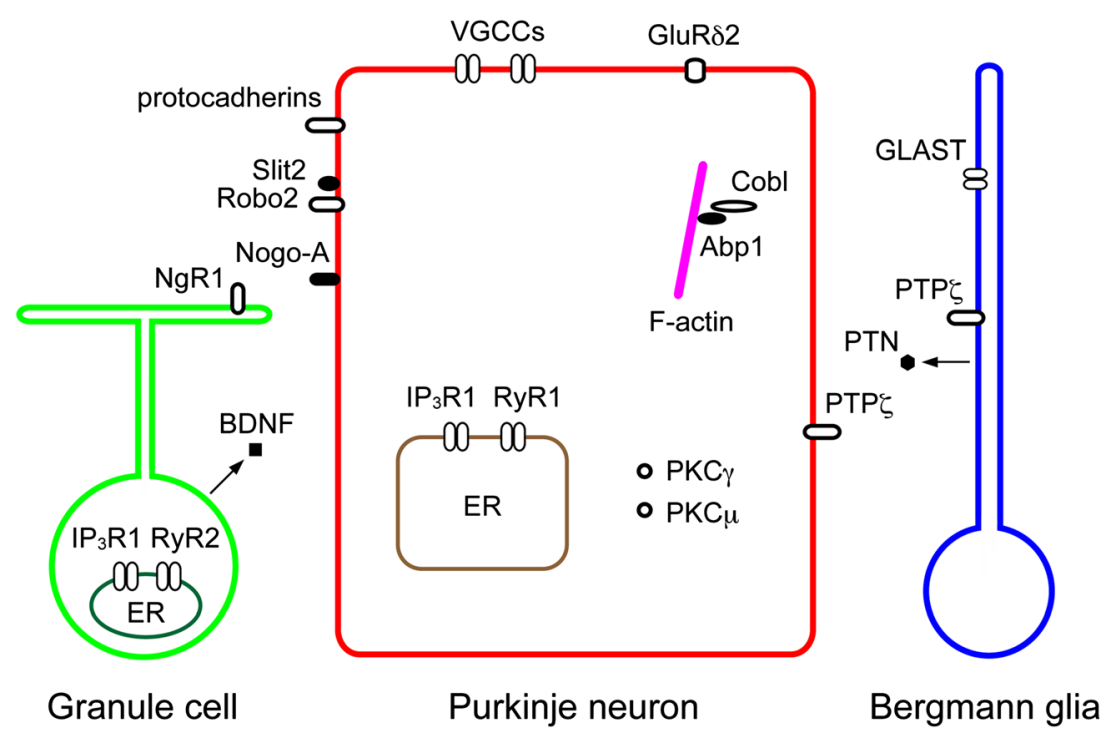


dendrites. Intracellular signaling mechanisms downstream of protocadherins and Robo 2 remain to be defined. It should be noted that self-avoidance mediated by Slit2/Robo 2 signaling occurs between high-order branches and not between primary dendrites [18]. Thus, mechanisms of retraction and selfavoidance may differ between such high-order branches and primary dendrites.

As another aspect of morphological changes of Purkinje dendrites, a recent three-dimensional confocal analysis demonstrated that dendrites of the majority of Purkinje neurons extend in multiple parasagittal planes in mice on postnatal day 18 [19]. The ratio of multiplanar Purkinje neurons decreased by postnatal day 25 , suggesting morphological changes from multi- to monoplanar. This remodeling of dendrites occurs in parallel to the refinement of climbing fiber inputs to Purkinje neurons and involves GluR $\delta 2$ and GLAST, a glutamate transporter expressed by Bergmann glia. It remains to be elucidated whether the retraction of minor planes actually occurs and how climbing fiber inputs, GluR $\delta 2$, and GLAST are associated with this remolding.

\section{Oriented Extension}

Another feature of Purkinje dendrites is that Purkinje neurons extend proximal dendrites in one direction, towards the pial surface. Although this is one of the intriguing problems in the morphogenesis of Purkinje dendrites, its molecular mechanism has remained a mystery.

A study using cerebellar slice cultures demonstrated that PTP $\zeta$ (RPTPß, Ptprz), a receptor-type protein tyrosine phosphatase synthesized as a chondroitin sulfate proteoglycan, and its ligand pleiotrophin (PTN) are involved in the oriented extension of Purkinje dendrites [12]. PTP $\zeta$ is expressed by Purkinje neurons and Bergmann glia, whereas PTN is expressed by Bergmann glia. PTN/PTP $\zeta$ signaling is required for the formation and/or maintenance of lamellate processes of Bergmann glia. Also, GLAST is involved in this mechanism downstream of PTP $\zeta$ signaling. Detailed mechanisms of downstream signaling of $\mathrm{PTP} \zeta$ remain to be elucidated. In addition, the secreted form of $\mathrm{PTP} \zeta$, phosphacan, may fulfill another function in the oriented extension of dendrites in the extracellular matrix around Purkinje neurons.

\section{Concluding Remarks}

Purkinje neurons form the most elaborate dendritic trees among neurons in the brain. Since Purkinje neurons are key elements in cerebellar functions, mechanisms of differentiation of their dendrites are important for understanding the relationships between cerebellar circuitry and functions. In addition, such mechanisms are intriguing from the viewpoint of the characteristic morphogenesis of cells. Although many studies have investigated extensive branching, and several recent studies have examined the retraction of dendrites, our understanding of the molecular mechanisms of these phenomena is far from complete. Furthermore, too little is known about mechanisms of oriented extension. It is notable that recent studies, as mentioned above, utilized superior techniques in molecular and cellular biology, such as knockout/knockdown, slice culture, three-dimensional morphological analysis, and live imaging. In addition, gene expression analysis and computational biology would support our investigation. Now, we anticipate excellent studies utilizing these techniques to clarify the molecular mechanisms governing the three important aspects: extensive branching, retraction of dendrites, and oriented extension, of the dendritic differentiation of Purkinje neurons.

Conflict of Interest The author declares no conflicts of interest in this submission.

\section{References}

1. Altman J, Bayer SA. Development of the cerebellar system: in relation to its evolution, structure, and functions. New York: CRC Press, Inc.; 1997.

2. Kapfhammer JP. Cellular and molecular control of dendritic growth and development of cerebellar Purkinje cells. Prog Histochem Cytochem. 2004;39:131-82.

3. Tanaka M. Dendrite formation of cerebellar Purkinje cells. Neurochem Res. 2009;34:2078-88.

4. Lohmann C, Wong ROL. Regulation of dendritic growth and plasticity by local and global calcium dynamics. Cell Calcium. 2005;37: 403-9.

5. Miyazaki T, Hashimoto K, Shin H-S, Kano M, Watanabe M. P/Qtype $\mathrm{Ca}^{2+}$ channel $\alpha 1 \mathrm{~A}$ regulates synaptic competition on developing cerebellar Purkinje cells. J Neurosci. 2004;24:1734-43.

6. Hisatsune C, Kuroda Y, Akagi T, Torashima T, Hirai H, Hashikawa T, et al. Inositol 1,4,5-trisphosphate receptor type 1 in granule cells, not in Purkinje cells, regulates the dendritic morphology of Purkinje cells through brain-derived neurotrophic factor production. J Neurosci. 2006;26:10916-24.

7. Ohashi R, Sakata S, Naito A, Hirashima N, Tanaka M. Dendritic differentiation of cerebellar Purkinje cells is promoted by ryanodine receptors expressed by Purkinje and granule cells. Dev Neurobiol. 2014:74:467-80.

8. Kessels MM, Schwintzer L, Schlobinski D, Qualmann B. Controlling actin cytoskeletal organization and dynamics during neuronal morphogenesis. Eur J Cell Biol. 2011;90:926-33.

9. Haag N, Schwintzer L, Ahuja R, Koch N, Grimm J, Heuer H, et al. The actin nucleator Cobl is crucial for Purkinje cell development and works in close conjunction with the F-actin binding protein Abp1. J Neurosci. 2012;32:17842-56.

10. Hendelman WJ, Aggerwal AS. The Purkinje neuron: I. a Golgi study of its development in the mouse and in culture. J Comp Neurol. 1980;193:1063-79.

11. Armengol J-A, Sotelo C. Early dendritic development of Purkinje cells in the rat cerebellum. A light and electron microscopic study using axonal tracing in 'in vitro' slices. Brain Res Dev Brain Res. 1991;64:95-114. 
12. Tanaka M, Maeda N, Noda M, Marunouchi T. A chondroitin sulfate proteoglycan PTP $/ /$ RPTP $\beta$ regulates the morphogenesis of Purkinje cell dendrites in the developing cerebellum. J Neurosci. 2003;23: 2804-14.

13. Lordkipanidze T, Dunaevsky A. Purkinje cell dendrites grow in alignment with Bergmann glia. Glia. 2005;51:229-34.

14. Tanaka M, Yanagawa Y, Obata K, Marunouchi T. Dendritic morphogenesis of cerebellar Purkinje cells through extension and retraction revealed by long-term tracking of living cells in vitro. Neuroscience. 2006; 141:663-74.

15. Fujishima K, Horie R, Mochizuki A, Kengaku M. Principles of branch dynamics governing shape characteristics of cerebellar Purkinje cell dendrites. Development. 2012;139:3442-55.
16. Petrinovic MM, Hourez R, Aloy EM, Dewarrat G, Gall D, Weinmann $\mathrm{O}$, et al. Neuronal Nogo-A negatively regulates dendritic morphology and synaptic transmission in the cerebellum. Proc Natl Acad Sci U S A. 2013;110:1083-8.

17. Lefebvre JL, Kostadinov D, Chen WV, Maniatis T, Sanes JR. Protocadherins mediate dendritic self-avoidance in the mammalian nervous system. Nature. 2012;488:517-23.

18. Gibson DA, Tymanskyj S, Yuan RC, Leung HC, Lefebvre JL, Sanes JR, et al. Dendrite self-avoidance requires cell-autonomous Slit/Robo signaling in cerebellar Purkinje cells. Neuron. 2014;81:1040-56.

19. Kaneko M, Yamaguchi K, Eiraku M, Sato M, Takata N, Kiyohara Y, et al. Remodeling of monoplanar Purkinje cell dendrites during cerebellar circuit formation. PLoS One. 2011;6:e20108. 